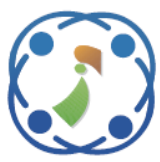

\title{
An Integrated System for the Seamless Localization and Specification of a Position Based on an Indoor-Outdoor Conditions in Ubiquitous Computing Environments
}

\author{
Khamla NonAlinsavath ${ }^{1,2,3 *}$ \\ Kazuhiko Hamamoto ${ }^{2}$ \\ Lukito Edi Nugroho ${ }^{1}$ \\ Widyawan $^{1}$ \\ Somphone Kanthavong ${ }^{3}$ \\ ${ }^{I}$ Department of Electrical Engineering and Information Technology, Faculty of Engineering, \\ Universitas Gadjah Mada, Yogyakarta, Indonesia \\ ${ }^{2}$ Department of Information Media Technology, School of Information and Telecommunication Engineering, \\ Tokai University, Tokyo, Japan \\ ${ }^{3}$ Department of Computer Engineering and Information Technology, Faculty of Engineering, \\ National University of Laos, Vientiane, Laos \\ * Corresponding author's Email: khamla@mail.ugm.ac.id
}

\begin{abstract}
The growing research interest in the localization tracking system has prompted development alongside availability positioning techniques to apply to the real environment and current situation. An integrated system that can support navigation and tracking in both indoor and outdoor conditions has been equipped with location information to improve the requirement and fulfil the demand of a seamless localization system based on existing technologies. This paper proposes a system called an integrated location-aware computing system using received signal strength indicator to support the direction and location information that works together with a smart devices and integrates an indoor-outdoor environment seamlessly by combining both conditions to be the system without any obstacle. Furthermore, the technologies of indoor and outdoor will be integrated into the users. Then, they move from different conditions; the system can provide as an indicator for the complication that arises between indoor and outdoor locations, as well as location-based service technology itself. The transition between indoor and outdoor is less than 5 seconds switching at the overlapped area, the certainty value of the performance when moving from outdoor to indoor is $98.4 \%$ accuracy, from indoor moved to outdoor is $97.7 \%$ accuracy at the first floor. Moreover, having this seamless location system makes a lot of benefits to assist the users in discovering interesting things from the system, enable the seamless in real-time operation based on Android platform and providing direction maps and multi-pathways from current locations to destinations, the combination between different environments is generated with higher performance and smoothly works when users have many alternatives to choose the available signals from both indoor and outdoor technologies, transmission computation of switching from indoor to outdoor and vice versa has been decreased time consumption with excellent reliability and continuity service by proposed system.
\end{abstract}

Keywords: Seamless location, Location tracking, Ubiquitous computing, Indoor/outdoor environment.

\section{Introduction}

At present, the popularization of technologies that integrate communication, computation, sensing, and processing into the physical world is being rapidly developed. Smart devices and localization technologies are becoming increasingly important, especially in smart computing systems that are aware of the environment and can analyze real-time situations. Location awareness is an example of a localization technique [1] in the field of ubiquitous computing, which makes use of location sensing in both indoor and outdoor environments. Ubiquitous computing [2] is a computing paradigm, where technologies become virtually invisible in our lives. The system enables interactions between human and physical devices throughout sensors and location 
technologies such as wi-fi fingerprinting [3], Bluetooth, and Global Positioning System (GPS). The intelligence in ubiquitous computing is mostly accomplished by context awareness that helps ubiquitous applications to sense contexts information around the environment and adapt to context types [4] that could change according to the situation.

The applications that can sense their physical and computational environment, such as the current location of the user, the relative location of people, hosts, accessible devices, network connectivity, and social situation, are called context-aware applications. These fall in the field of ubiquitous computing, while context-awareness provides computing environment with the ability to adapt to the services or information being provided. It is an ability to sense and derive the user's demand automatically, which separates the context-aware applications from traditionally designed applications to make them more attentive, responsive, and aware of the user's identity and the user's environment. A context-aware application [5] is defined as the ability of the program or computing devices that can be sensed, acted, or interpreted in the context of an entity to modify its behaviors to meet the context user.

Nevertheless, some issues arise when applying the system to the real environment due to the complexity of integrating both hardware and software in such technologies. The accuracy of the system is also inadequate to apply to the pervasive atmosphere. There is no single location technology that is wholly accurate, low-cost, and easy to deploy, as location tracking context encounters the fusion of multi-layers of scoping context for integrating many locations and positioning systems. There are many location technologies [6], each with its challenges that should be improved to enhance further the accuracy required by the location application.

Indoor positioning tracking and navigation systems [7] measure and display total coverage area in the specific places based on the internal environment only. The map also offers location computing system in the current situation using indoor technologies such as wi-fi, and iBeacon. Furthermore, outdoor localization uses a GPS technology to provide location information in terms of an outdoor environment. GPS is usually equipped with a smartphone and low power sensors that provide surrounding information and the movement tracking such current location information, user's information, and positioning.

The integration of outdoor-indoor environment (overlapped area) [8] can lead to the development of a rich knowledge-based technology. These are not only useful for social services, but also a good input into a system based on the individual habits because they can optimize user's daily routines. The technologies for seamlessness of location are essential to achieve the requirement of unified connectivity when the user is moving between different conditions. The complexity between multilayers of location context taxonomy needs to be considered as location granularity of outdoor and indoor conditions are to be integrated to the system seamlessly. Besides, the transmission of location data at different levels of granularity generally requires a location-aware technique that is agreed upon by both services. The technique will assist the system of location-aware applications to operate better in sensing the location. Moreover, the complex location model needs to be improved for the users across the boundary between different levels of geography such as country, state, province, and region.

Furthermore, users will be able to analyse and understand the different levels of context scoping. Navigation and tracking of location-based services are also needed to improve some issues that still exist according to the reviews of positioning technologies from quality of positioning services. These include response in real-time, accuracy with a few meters, seamless availability (indoors and outdoors), excellent reliability and continuity of service, lowmedium power consumption, reasonable or cheap price and technical standards for protection of the data [10].

In this research, a location technique that was modeled based on the scenario of the system was proposed to validate the efficiency of the navigation system and location awareness to provide the user's direction and specific positioning when the user moves from different environments. This work was extended from our own previous work [11] which is focused on an indoor localization and specification based on Wi-Fi fingerprint, Bluetooth low energy and pedometer. Therefore, this work emphasizes on an integration or seamlessness between indoor and outdoor conditions to localize and track the positioning when user moves from outdoor environment to overlapped until specific positioning of indoor region. The purpose of the seamless solution is to combine the indoor and outdoor conditions to be a completed system seamlessly based on received signal strength indicator (RSSI) to detect and locate the current location and to be aware that user is in indoor or outdoor regions. The technologies of the indoor and outdoor were integrated to provide all location scenarios refer with Table 1 provides the different works as an indicator that a proposed method can be a solution for the 
complication that was raised between indoor and outdoor locations.

This paper is organized into several sections: section 2 presents the state of the art as well as related works for seamless localization; section 3 presents the system implementation and experiment setup for an indoor and outdoor positioning system; section 4 figures out the results of the experiment based on indoor and outdoor location system; which finalize the seamlessness of both conditions together; conclusion is provided in section 5 .

\section{Related works}

Numerous researchers conducted location awareness systems with specific aims to overcome the perspective and identify different issues. Their previous studies have provided different methods to the system in each condition environment; they developed the system by their scenarios, objectives, methods, and outcomes. The previous related works based on seamless location have been presented using several technologies to integrate indoor and outdoor technologies to become a completed system such as GPS, Global System for Mobile Communication (GSM), and Ultra-wideband (UWB). To explore a qualified conceptual, simple, and more understandable location system based on indoor and outdoor environment, the previous works are summarized as below:

In [12] proposed a seamless pedestrian navigation methodology optimized for indoor/outdoor detection based on the smartphone fusion location method. Several signals such as light sensor signal, the magnetic sensor signal, and Global Navigation Satellite System (GNSS) signal were integrated into the navigation algorithm to improve the accuracy of the location identification. The seamless position system was organized on an Android intelligent terminal, and an indoor map was added to the Baidu Map. The indoor/outdoor detection result relied on the comprehensive judgment of the light sensor, the magnetic sensor, and the GNSS. The accuracy of the indoor environment increased by 55\%; this strategy was beneficial to improve the overall accuracy of the positioning.

In another study [13] proposed the issue of distributed localization based on an integration of a specific set of radio measurements, the problem is studied in detail from a signal processing point of view in its most feneral and widely used statistic form. The identified solutions are analyzed from both computational complexity and localization accuracy perspectives, with a theoretical lower bound included in the latter comparison in order to assess the margin available for future improvement. received signal strength (RSS) and angel of arrival (AOA) measurements are distributed and investigated in this study, an analysis from both computation complexity and localization accuracy was provided and calculated in term of the performance of existing approaches to give an intuition that used theoretical lower bound. Analysis of computation complexity is achieved by considering a fully connect network, for instance, the total number of neighbors of each target is assumed to be $\left(\left|\varepsilon_{i}\right|=N+(M-1), \quad \forall_{i} \in \tau\right)$. Several algorithms are provided to analyze the performance such as SOCP (second-order cone programming), LLS (linear least squares), MCMC-MH (Markov chain monte carlo with metropolis-hasting) and GTRS (generalized trust region sub-problem. An averages execution time of each algorithm are given as following: SOCP in complexity of $O\left(\left|\varepsilon_{i}\right|^{3.5}\right)$ with execution time 0.66 seconds (sec), LSS in complexity of $O\left(\left|\varepsilon_{i}\right|\right)$ with execution time $0.004 \mathrm{sec}$, MCMC$\mathrm{MH}$ in complexity $O\left(C_{\max } \times T_{\max } \times\left|\varepsilon_{i}\right|\right)$ is $1.2 \mathrm{sec}$ execution time, and GTRS $O\left(S_{\max } \times\left|\varepsilon_{i}\right|\right)$ with execution time $0.005 \mathrm{sec}$. Analysis of localization accuracy has been generated through RSS and AOS measurements that can be summarized as following: the network is connected and does not vary during the computation period, all nodes are suitably equipped to measure RSS of the received radio signal, some node or possibly all of them are conveniently equipped to measure $\mathrm{AOA}$ of the received radio signal, working order of the nodes is synchronized.

Similarly, [14] proposed a collation between performance investigations of a location-based routing protocol geocast adaptive mest environment for routing (GAMER) with contaxtual information collected from global positioning system (GPS) and framework for internal navigation and discovery (FIND), the systems are evaluated based on various metric such as accuracy, packet delivery ratio and packet overhead by mean of network simulator. Framework for internal navigation and discovery can be used as an information source for operations performed through the data collected by motion sensor and GPS, it provides indoor positioning making the overall system more accurate than systems mentioned, the geocast data acquired through FIND may be used from minimal lifestyle tracking and household automation to extensive commercial applications. The evaluation discloses that FIND based GAMER performs better in most cases, result obtained are better than GPS in both indoor and outdoor environment with mobility, accuracy of FIND is below $10 \mathrm{sq}$. $\mathrm{ft}$. as compared to GPS which has an accuracy up to a few meters. The performance of both systems declines as compared 
node system, packet delivery ratio of FIND based GAMER remains above $90 \%$ for most case, but GPS based GAMER loses the performance when the node speed surpasses $10 \mathrm{~m} / \mathrm{sec}$., the performance of the framework for internal navigation and discovery system remains superior to global positioning system in all aspects.

Besides, [15] introduced an approach for hybrid indoor/outdoor navigation to integrate the indoor and outdoor and to provide a seamless hybrid navigation experience. Model to capture indoor environments and localization algorithm of a new service that support integrated indoor and outdoor routing was also proposed. First, the authors described the indoor modeling that was used to represent indoor environments, while its application for indoor routing, approach localization, and integration issues were described as the last part. The services were extended into maps, places, and direction to integrate the indoor model, routing, and localization into the outdoor infrastructure. Maps provided the tile-based interface with an optional parameter to specify the

Table 1. Comparison the different between existing works and proposed system

\begin{tabular}{|c|c|c|c|}
\hline No & Authors & Methods & Result and Accuracy \\
\hline 1 & $\begin{array}{l}\text { Q. Zeng, J. Wang, Q. } \\
\text { Meng, X. Zhang, and } \\
\text { S. Zeng [17] }\end{array}$ & $\begin{array}{l}\text { Provided light, magnetic and GNSS } \\
\text { sensor signals that integrated to } \\
\text { navigate }\end{array}$ & $\begin{array}{l}\text { The experiment results indicate that the } \\
\text { continuity and the accuracy if the seamless } \\
\text { position between the two environments are } \\
\text { effectively improved, The accuracy of the } \\
\text { indoor increased by } 55 \% \text {. }\end{array}$ \\
\hline 2 & $\begin{array}{l}\text { W. Jiang, Y. Li, C. } \\
\text { Rizos, B. Cai, and W. } \\
\text { Shangguan [18] }\end{array}$ & $\begin{array}{l}\text { Locata GNSS and INS are proposed to } \\
\text { implement. The federated Kalman } \\
\text { filtering as data fusion algorithm }\end{array}$ & $\begin{array}{l}\text { Able to provide seamless indoor-outdoor with } \\
\text { 3D accuracy better than } 5 \mathrm{~m} \text {. } \\
\text { System has better performance in term of } \\
\text { positioning and determination }\end{array}$ \\
\hline 3 & $\begin{array}{l}\text { Y. Gao, S. Liu, M. M. } \\
\text { Atia, and A. Noureldin } \\
{[19]}\end{array}$ & $\begin{array}{l}\text { GPS, INS and LiDAR are integrated, } \\
\text { Hybrid Scan Matching algorithm }\end{array}$ & $\begin{array}{l}\text { Sub-meter navigation accuracy and accurate } \\
\text { attitude angles estimation can be accomplished } \\
\text { during the whole } 99.83 \% \text { of the trajectory } \\
\text { including outdoor and indoor environments }\end{array}$ \\
\hline 4 & $\begin{array}{l}\text { Eyng. A, Rayel. O, } \\
\text { Oroski. E, Rebelatto, J } \\
{[20]}\end{array}$ & $\begin{array}{l}\text { The Bluetooth low energy is proposed } \\
\text { a hybrid indoor positioning system } \\
\text { both multilateration and fingerprint } \\
\text { RSSI-based, also adopted Kalman } \\
\text { filtering (KF) along with track to track } \\
\text { fusion (TTF) }\end{array}$ & $\begin{array}{l}\text { The result indicates that the proposed system } \\
\text { improves the estimation accuracy when } \\
\text { individually compared to the standalone } \\
\text { fingerprint scheme in up to } 46 \% \text {, while the } \\
\text { standalone multilateration is outperformed in } \\
\text { approximately } 54 \% \text {. The probability that the } \\
\text { distance error of the proposed hybrid indoor } \\
\text { positioning system is lower than } 2 \text { meters is } \\
92 \% \text {, while fingerprint is } 43 \% \text { and } \\
\text { multilateration is } 47 \% \text { accuracy. }\end{array}$ \\
\hline 5 & $\begin{array}{l}\text { Kazikli. E, Gezici. S } \\
\text { [21] }\end{array}$ & $\begin{array}{l}\text { TDOA (time different of arrival) and } \\
\text { RSS (received signal strength) } \\
\text { estimates are proposed for the first } \\
\text { step and position estimation, two step } \\
\text { positioning technique is shown to } \\
\text { direct positioning at high signal to } \\
\text { noise ratios based on asymptotic } \\
\text { propertied of maximum likelihood } \\
\text { (ML) estimation. }\end{array}$ & $\begin{array}{l}\text { The result has been observed that in the low } \\
\text { signal to noise ratio regime, the information } \\
\text { carried in the time information is erroneous, } \\
\text { distorting the overall performance of the two- } \\
\text { step approach is more convenient at high SNRs } \\
\text { due to its computational efficiency while the } \\
\text { direct approach is more preferable in the low } \\
\text { SNR regime due to its improved performance. }\end{array}$ \\
\hline 6 & Proposed System & $\begin{array}{l}\text { Proposed integrated system based } \\
\text { indoor using Wi-Fi, BLE and } \\
\text { Pedometer and Outdoor using GSM } \\
\text { (LTE) and GPS based on received } \\
\text { signal strength indicator algorithm, } \\
\text { the integration of environments } \\
\text { (indoor and outdoor regions), and } \\
\text { integration between indoor and } \\
\text { outdoor technologies to provide all } \\
\text { locations scenarios and indicate a } \\
\text { solution for complication based on } \\
\text { localization technologies. }\end{array}$ & $\begin{array}{l}\text { Completely accomplished the integration of } \\
\text { Wi-Fi, BLE, Pedometer, GSM (LTE) and GPS } \\
\text { that work together, transaction uses a single } \\
\text { system, seamless in real-time operation that } \\
\text { provide direction and location information } \\
\text { seamlessly by } 98.4 \% \text { of certainty value from } \\
\text { outdoor to indoor region, and from indoor } \\
\text { moved to outdoor is } 97.7 \% \text { accuracy and less } \\
\text { than } 5 \text { seconds transition time between indoor } \\
\text { to outdoor regions. Seamless tracking shows } \\
\text { the exact positioning of the user when he/she } \\
\text { moves from one position to destination with } \\
\text { continuously service in seconds of switching. }\end{array}$ \\
\hline
\end{tabular}


level, while a mobile device indicated a combined visualization of an indoor and outdoor environment by drawing the indoor tile on top of the outdoor plan. Places provided the location model of the address to capture the level of the location and integrate the indoor places into the place service. Directions extended the interface of the directions service with an optional level for the route origin and destination. Furthermore, the system has been implemented over a mobile application for Android and ios that supports hybrid navigation by using the proposed infrastructure. The application can compute multimodal routes using various public transport options available in Madrid and Moncloa that enable indoor navigation as a part of the trip. In another study, [16] proposed that a smartphone seamless positioning system based in outdoor and indoor mobile navigation electronic map connected between indoor and outdoor geographic information, and convenient for initial sensors and GNSS module. The Baidu map was used to carry on the secondary development to support the solution and display in order to improve the availability and autonomy of pedestrian navigation system in the indoor environment. The Baidu Map API, positioning API and layer custom API consisted of many technologies such as autonomous inertial navigation technology, inertial navigation, magnetic sensors, satellite navigation, and other cooperative working mechanisms. The walking experiment started outdoors and ended indoors The GNSS signal was blocked not only indoors, but also blocked by buildings and trees outdoors. It can be seen that the unit of the horizontal coordinate reflects the pedestrian trajectory well with navigation of two-dimensional position error at starting points of 4.69 meters. The whole navigation results reflected the walking track very well, with the real-time display and redisplay of the pedestrian trajectory.

\section{Implementation and experiment setup}

\subsection{System design and implementation}

The system can provide continuous positioning solutions with existing outdoor applications like Google maps based on GPS and indoor technologies due to integrating both scenarios to be merged as one complete system. The proposed system of a seamless indoor-outdoor approach can improve the performance of positioning and navigation application. The proposed system focuses on the combination of indoor and outdoor technologies to become the direction for the users, together with integrating the environment conditions seamlessly.

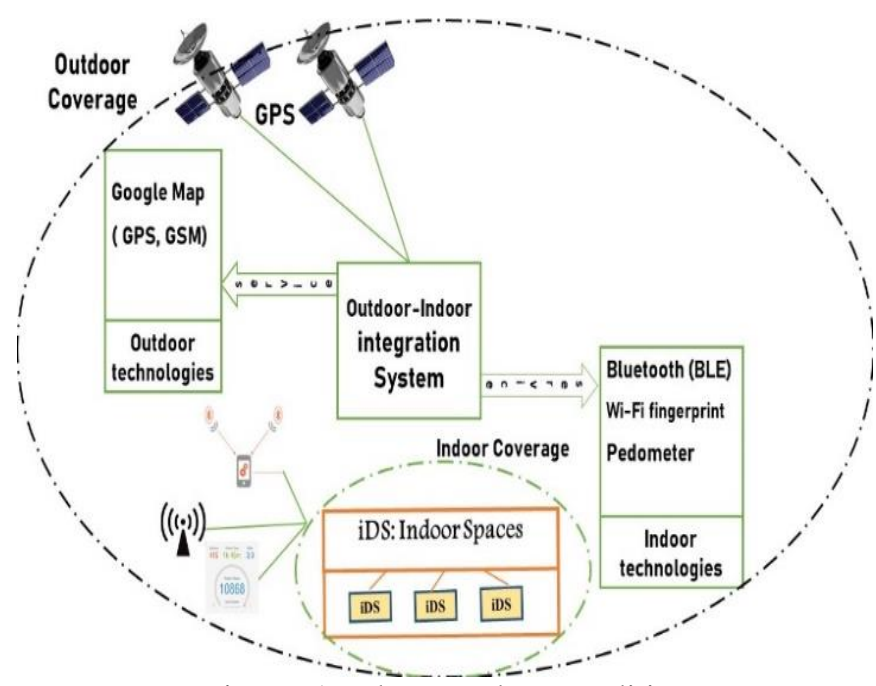

Figure. 1 Indoor-outdoor conditions

The deployment of a seamless conceptual framework in Fig. 1 for application response services like navigating, positioning, and routing awareness is advance and beyond the solution of the existing heterogeneous combination in global and local based localization systems. The seamless routing to the network connection for the user is considered; it will transfer information by swapping the technology of indoor and outdoor environments with regards to the user moving in or out from the overlapped positioning and the different actual localization.

An experiment of the proposed system in this research was conducted in an outdoor and indoor environment with the maximum accuracy results. The system can fulfil the requirements of the positioning system based on indoor and outdoor localization. The system can also be applied to various situations and multiple devices with the integrated system in high quality of results and services.

The proposed modelling mentioned has been developed and defined as implementing into indoor and outdoor environments seamlessly. The main application of localization for indoor and outdoor is expected to assist living comfortably and become an important role system that can cover the challenge of human life.

The potential of this system is to change from outdoor to indoor techniques automatically once the users move to the border between indoor and outdoor boundary. They do not need to swap the application into indoor applications while they are performing or directing from outdoor positioning. The globally indoor and locally outdoor boundary will be separated, and both of them work differently to measure the target's location accurately. The distance between outdoor and indoor will be solved and connected seamlessly. At the same time, the users 


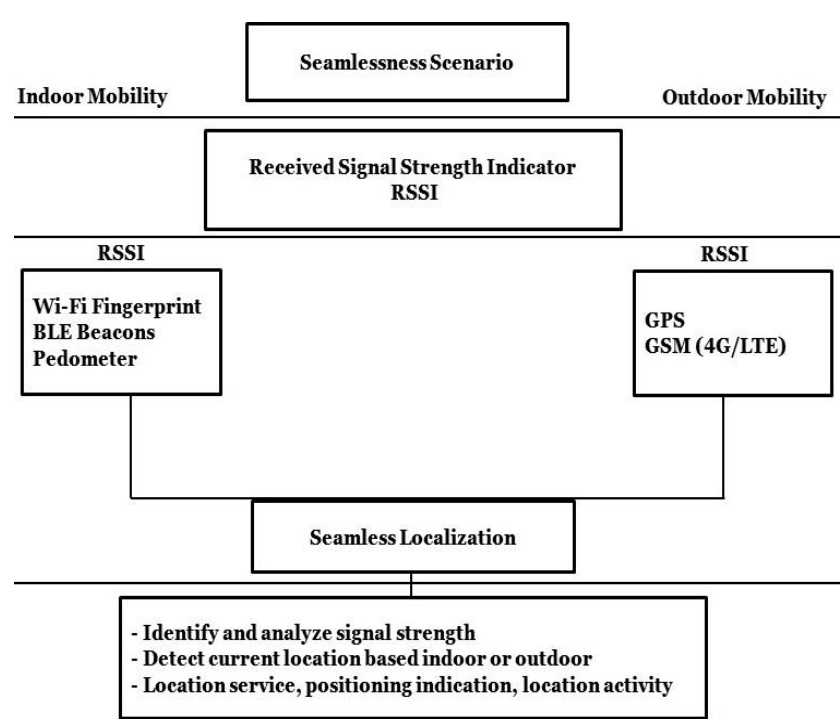

Figure. 2 Seamless indoor and outdoor scenario and framework

have to navigate to the specific point of indoor location from the large size of scale or deployment area as well as globally to locally particular positioning.

The proposed application of positioning in Fig. 2 provides beneficial and useful methods to the location and navigation based on indoor-outdoor environment continually. The system is called integration of an indoor-outdoor environment with GPS, wi-fi fingerprinting, bluetooth and pedometer; these have been proposed by combining all of the technologies to perform as a real-time application with a high accuracy to monitor the real situation. Finally, the system is integrated between indoor and outdoor conditions seamlessly.

\subsection{Experiment setup}

Various positioning technologies implementation are approached to locate and integrate location based on indoor-outdoor conditions. In this research, received signal strength indication technique as the fingerprinting method was utilized for the seamlessness of the localization and integrated wi-fi fingerprinting, bluetooth low energy beacons, pedometer, GPS, and GSM networks together.

Installation testing was one of the most significant parts of this experiment to ensure that the user and system did not face any difficulties while performing the application. In contrast, testing was aimed to verify the successful installation and updated situation when the user operates the application

We propose the seamlessness location system based on an Android application platform that were able to operate in both outdoor and indoor conditions

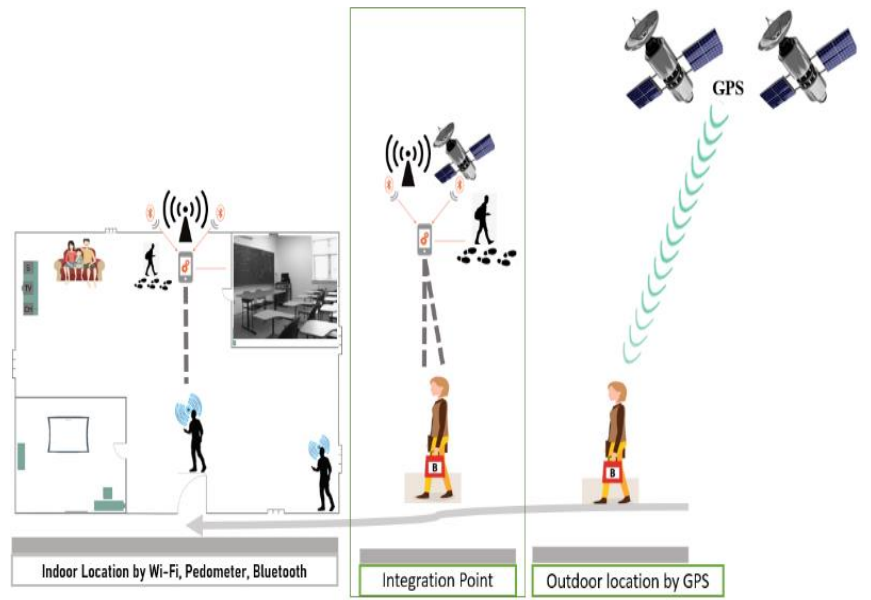

(a)

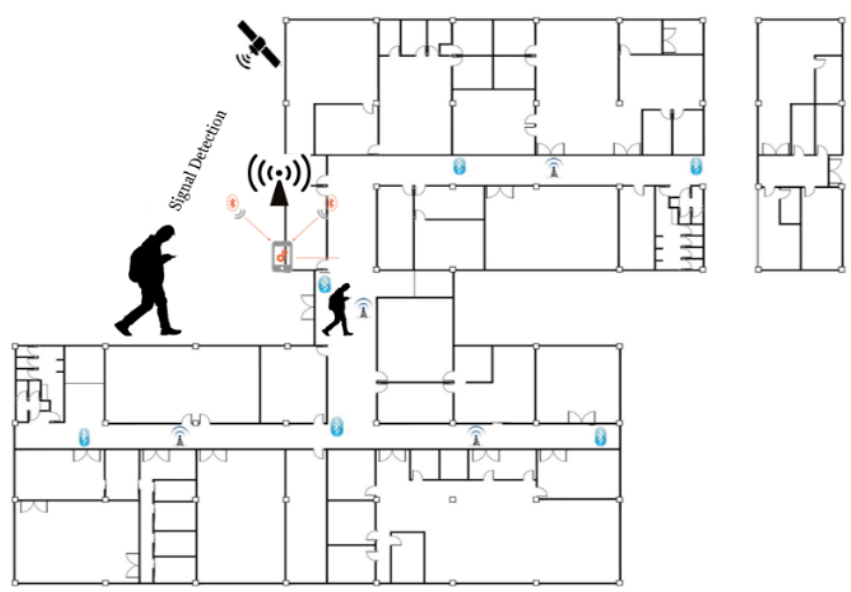

(b)

Figure. 3: (a) Seamless localization system for the indoor and outdoor environment and (b) experiment setup at campus building of the first floor for location tracking

continuously when users are moving from outdoor environment to indoors by detecting the available signal at the boundary between buildings and outdoor spaces. Then, users can get into the indoor directions without any obstacle or asking anyone else for direction guidance. The overlapped areas were considered as the main concentration of seamless localization in indoor and outdoor environments. Indoor localization used wi-fi fingerprint, Bluetooth low energy beacons and pedometer to classify and locate current positioning. These are the leading technologies in locating around building, while GPS and GSM were used in term of outdoor condition to estimate and track at the current region of the user. Signal strength measurement was applied to the system in order to be aware of whether the user is in indoor or outdoor environments by receiving the strongest signal at the moment. Log-Distance Path Loss [22] is applied as model formula to solve and conduct to the proposed system. 

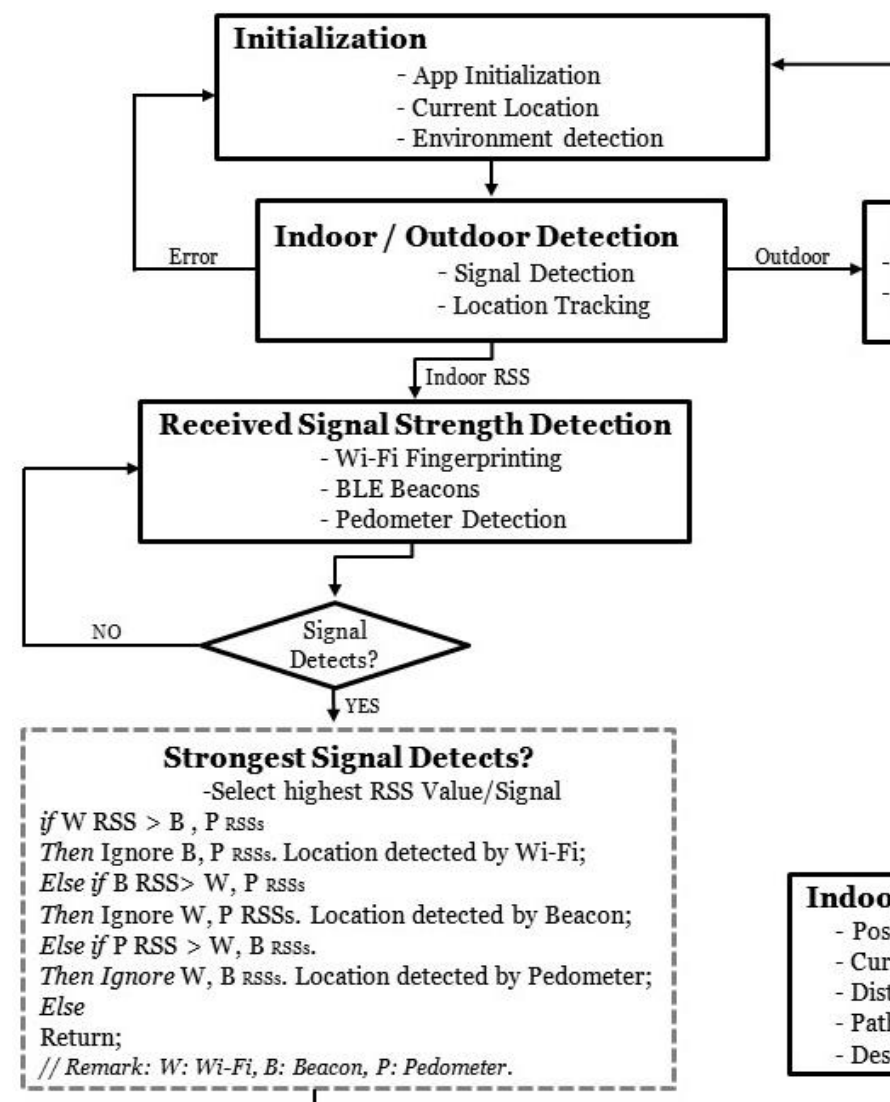

Received Signal Strength Detection

Global Positioning System (GPS)

Global System for Mobile Communications (3G, 4G / LTE)

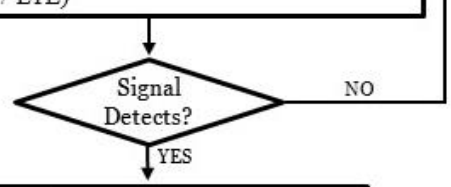
Outdoor Map Data:
- Positioning Data
- Current Location Data
- Location Activity
- Pathway / Direction
- Destination Data

Figure. 4 Seamless localization flow diagram and detection procedure

An integrated location-based on indoor and outdoor environments defines the RSSI (received signal strength indicator) value from provided technologies, including the signal of GPS, wi-fi signal, BLE (Bluetooth low energy) beacons, and pedometer, as shown in Fig. 4. The signals can be easily received by the user's smartphone between indoor and outdoor conditions. When the process detected the strongest signal from the current region, it also checked the signal changes for a seamless location at this time. Then, the process decided whether the received signal established connectivity between signal and users' smartphones.

The connection between indoor and outdoor environments integrated seamlessly in the overlapped regions as well as users' current positioning without any changes of the system and switch to other application., While users were moving to any direction of the building, the system analysed and detected a signal of validation. After that, users moved to the desired destination together with indoor location data.

$$
\operatorname{RSSI}(d)=\operatorname{RSSI}\left(d_{0}\right)-10 \times n \times \log \left(\frac{d}{d_{0}}\right)
$$

RSSI(d) is the received signal strength at distance $\mathrm{d}$ (between user at current location (receiver) and emitter). RSSI $\left(d_{0}\right)$ is the received signal strength at distance $d_{0}\left(d_{0}=1\right.$ meter $)$ and $\mathrm{n}$ is the path loss on the propagation environment.

Solving the Eq. (1) for distance $\mathrm{d}$ by applying $d_{0}$ $=1$ meter , the equation is simplified to:

$$
d=10 \frac{\operatorname{RSSI}\left(d_{0}\right)-\operatorname{RSSI}(d)}{10 \times n}
$$

As we know that $\mathrm{n}$ is parameter that depends on the transmission medium, the value of $d$ will also depend on these factors in order to find the value between current location (smartphone placement) and emitter, therefore the distance of path loss and signal strength measurement can be estimates as following equation:

$$
n=\frac{R S S I\left(d_{0}\right)-R S S I(d)}{10 \times \log \left(\frac{d}{d_{0}}\right)}
$$

The seamless localization routine performed the continuity service of positioning when users' movements were tracked from outdoor to indoor regions as shown in Fig. 1. The seamless function is operated if the user is moving to the destination by holding a smartphone that continuously gets the location data such as distance between two different 
positioning, indoor location data, pathway, and direction. The smartphone (system) checked whether the user entered the indoor region or not by comparing the received signal strength value between proposed technologies and available signals. If the current location is estimated and calculated by the previous positioning, then the system can assume that the current location is an indoor region. Both outdoor and indoor maps were provided, including a neighbour map of a coverage region.

To calculate the distance between three signals (Wi-Fi, BLE and GPS) that available and detected from user will be relied on this following technique:

$$
\begin{aligned}
& d_{1}^{2}=\left(x_{1}-x\right)^{2}+\left(y_{1}-y\right)^{2}+\left(z_{1}-z\right)^{2} \\
& d_{2}^{2}=\left(x_{2}-x\right)^{2}+\left(y_{2}-y\right)^{2}+\left(z_{2}-z\right)^{2} \\
& d_{3}^{2}=\left(x_{3}-x\right)^{2}+\left(y_{3}-y\right)^{2}+\left(z_{3}-z\right)^{2}
\end{aligned}
$$

The proposed localization tracking system for integrating both indoor and outdoor environments consisted of three sub-components, such as indoor, overlapped, and outdoor conditions, which used signal strength analysis and mapping building. Wi-fi fingerprint was not the only core technology that was used for indoor positioning in this work, as there were Bluetooth low energy beacons and a pedometer that were proposed to this system in order to have available alternative signals for the user when they cannot find other signals. The system collected signal strength of multiple different devices such as access points and beacons at a pending area. The process performed all the procedures; when the average signal strength value was calculated, then the system showed location information such as indoor map, pathway, and direction. In terms of overlapped environment, there was an area between indoor and outdoor coverage areas, which system detected all available signals., Then, the system can be described as a global indoor location and local outdoor location. At this integration point, the function configuration established and manages switch from outdoor to indoor condition seamlessly. We utilized google map platform for outdoor location by connecting to GPS or GSM signals for outdoor positioning. It was used for detecting the current position of longitude, and latitude of coordinates points

\section{The result of experiment and discussion}

We developed a testbed system based on an Android platform to evaluate the performance of the proposed system mentioned above. The proposed system was programmed as an Android application (Fig. 5) running on Samsung Galaxy S7 Edge and integrated between outdoor and indoor environments to combine both technologies scenarios such as GPS, GSM, Wi-Fi fingerprint, BLE beacons, and pedometer. In the meantime, the localization information, including the distance between two different points, indoor-outdoor mapping, pathway, and distance estimation were also attached to the system. The experimentation results were executed using a developed prototype in the provided indoor and outdoor regions by walking around the building from the outdoor condition. The system had been tested and applied to the Department of Electrical Engineering and Information Technology, Faculty of Engineering, Universitas Gadjah Mada. The performance of the developed system was evaluated by three measurement metrics such as GPS/GSM signal measurement as an outdoor region, GPS/GSM/wi-fi/beacons/pedometer as an overlapped region and wi-fi/beacons/pedometer as the indoor environment.

The testing on GPS/GSM had been performed in front of the building at a length of 15 meters to the overlapped area. An outdoor localization navigated from current location to destination with the detail of pathway and distance., The current localization of the user was identified and reached the selected location by the proposed application while the user was holding a smartphone. Then, the location was mapped with recorded value on the system with the corresponding positioning during the navigation period.

While user was moving to the overlapped region to test GPS/GSM/wi-fi/beacons/pedometer, the system started to listen and read from user's device that already generated the map., The location tracking had a different value of signal strength during the movement of user. The system indicated the current location that was detected from the highest value of the signal at the meantime. In this situation, the system detected all of the available signals around the building. The values of the signal were calculated and applied to every assigned point, and placement location of the devices. However, the transaction between outdoor into indoor condition took some time to calculate and to switch from one to another status based on received signal strength.

The current location was detected by the proposed application (Fig. 6) when the user moved into indoor localization. After that, the system continuously tracked and located an indoor positioning using wi-fi/beacons/pedometer. The transition time to switch the evironment of outdoor to indoor condition is less than 5 seconds, while the user is arriving the overlapped area, the transition will be classified and measured by signal strenght of 


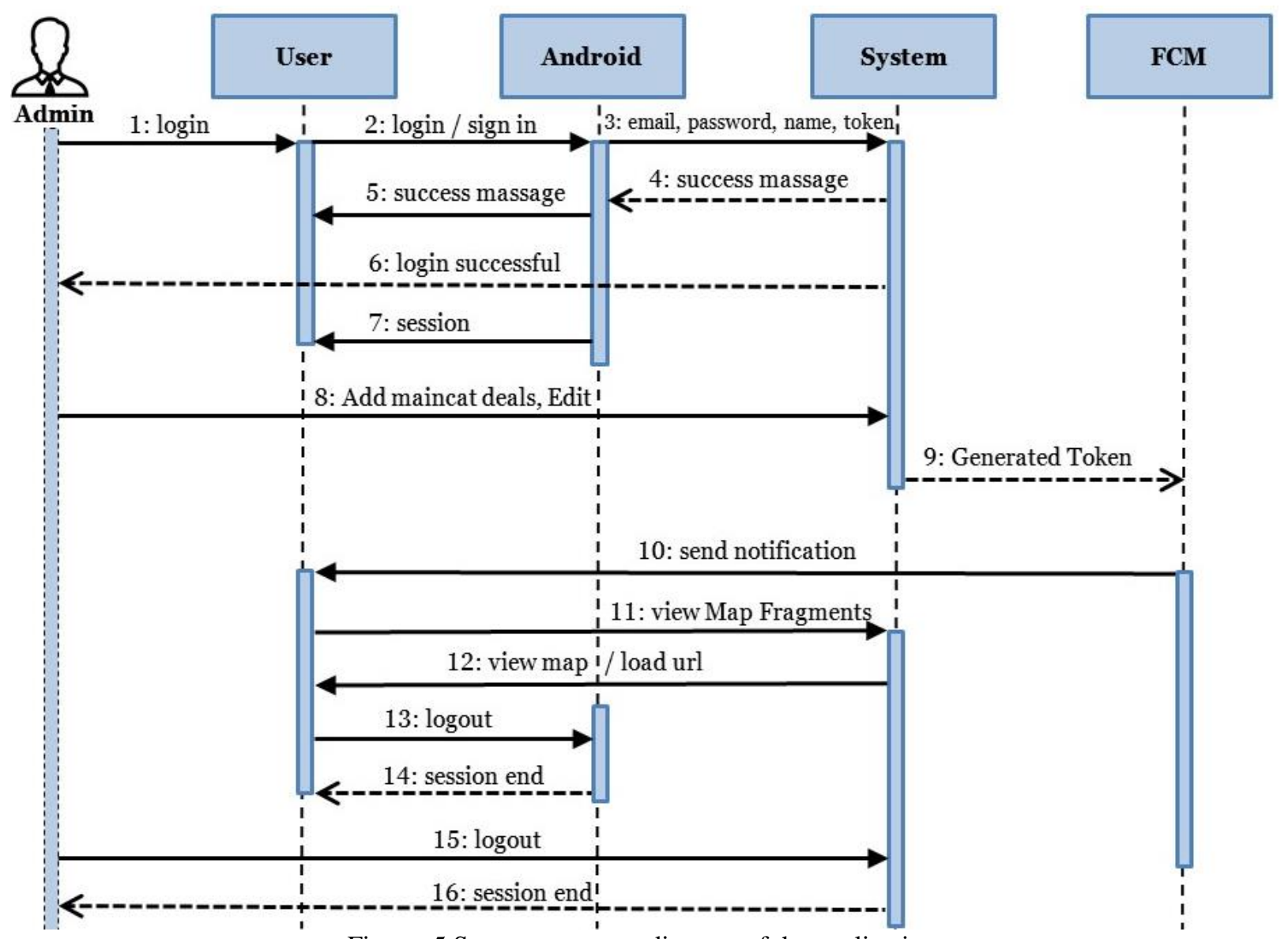

Figure. 5 System sequence diagram of the application

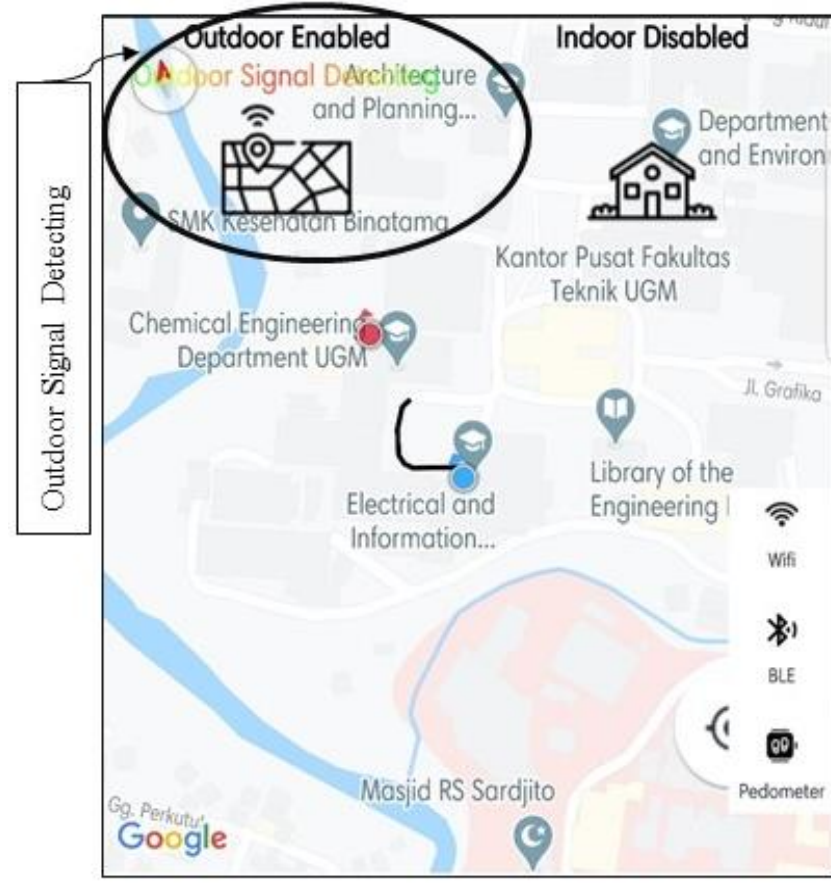

(a)

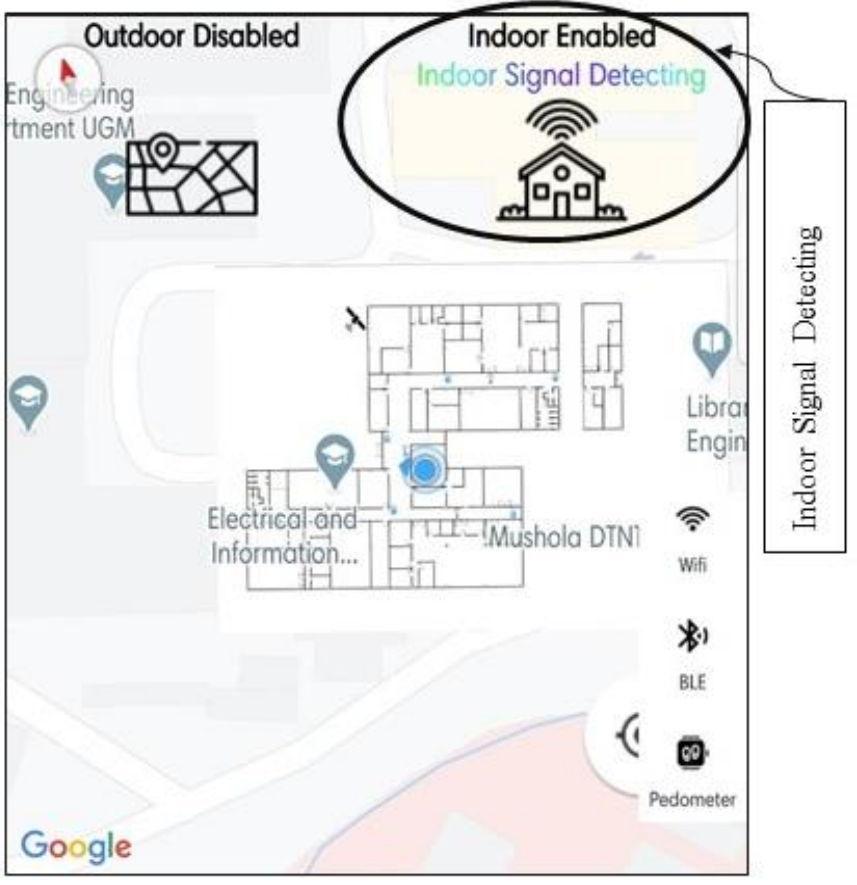

(b)

Figure. 6 Location detecting based on: (a) indoor and (b) outdoor conditions

received data that available indoor and outdoor localization, navigation and tracling processes are obtained important changes of the current positioning in few meters. In these three kinds of indoor technologies, one of the highest signal values were picked to be the navigation guidance, while the steps 


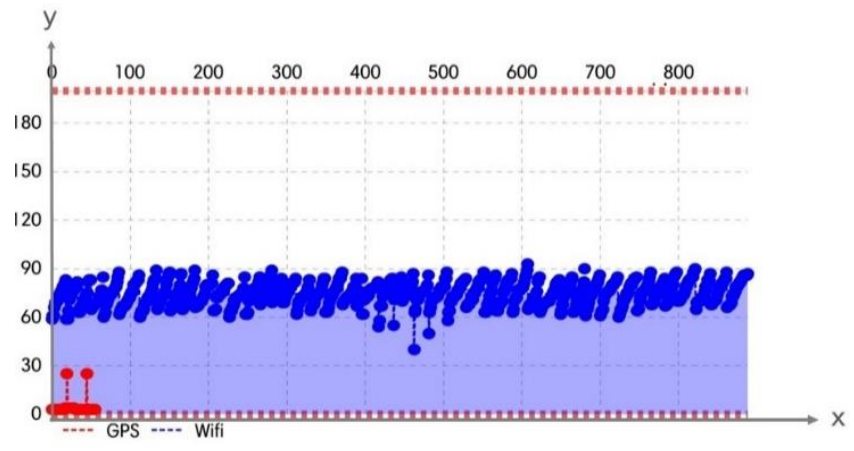

(a)

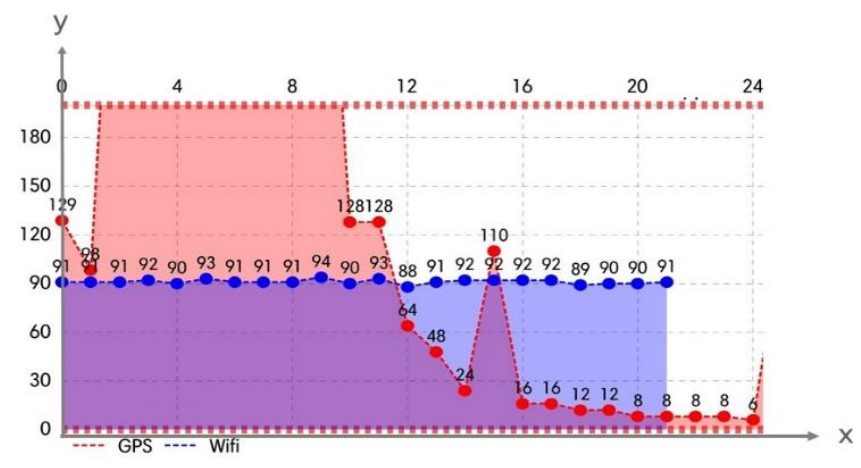

(b)

Figure. 7: (a) Outdoor detection based on GPS and (b) indoor detection based on Wi-Fi signal

of the users were counted until the user reached the destination. The experiment of the indoor region was performed at the selected reference points that were assigned by the developer based on the current positioning of longitude and latitude coordinate points. The walking patterns of the user around the building were recorded and identified into the database of the system to classify the different ways of the user's movements. The location and positioning were identified by using these scenarios to represent the layout of the test area. The blue dot represented current positioning point of the user, while an indoor map location served as regions when the user hit the recorded path positions. Then, the system detected the indoor region, went into the indoor navigation and notified additional information such as floor path and certainty level., As soon as the user exit from the indoor recorded position, the system notified and went to the outdoor tracking respectively.

On the seamless tracking process, the real-time access pointed to wi-fi fingerprint (Fig. 7), while bluetooth low energy beacons, pedometer signals and GPS location coordinates were integrated and tracked with the additional factors. For example, GPS coordinates were merged indoors, integration points, and outdoor conditions. The proposed devices served as a media at the center point in the indoor position

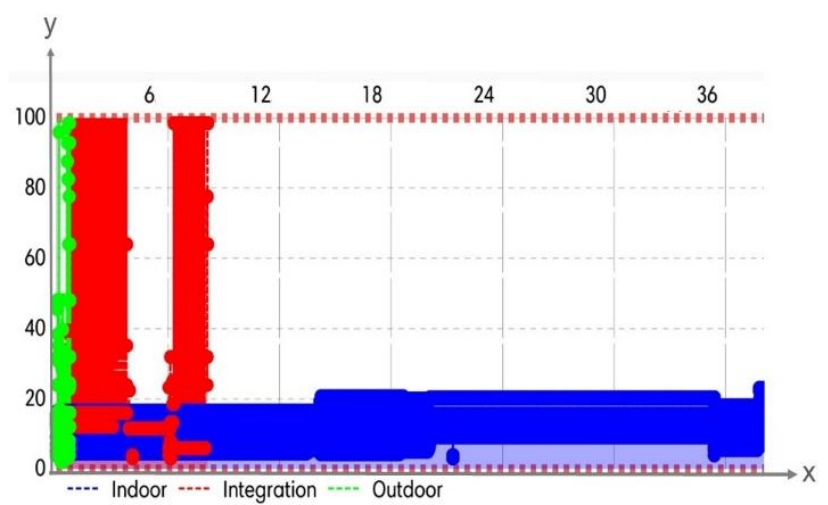

(a)

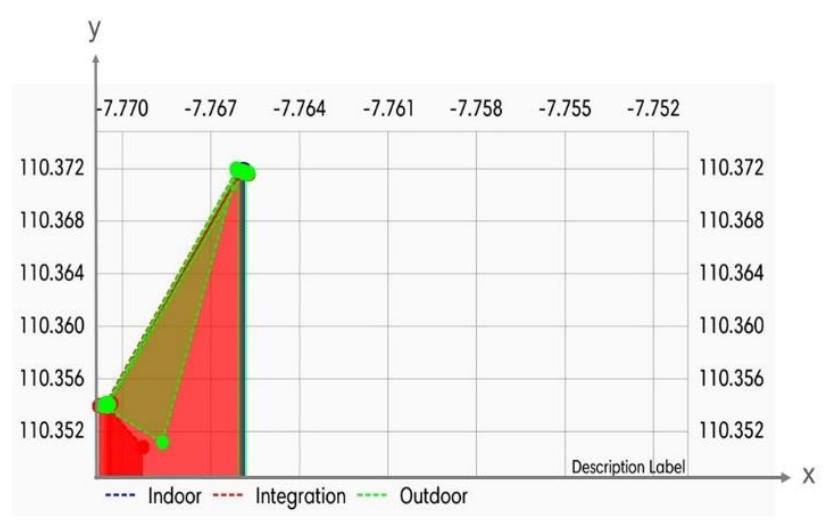

(b)

Figure. 8: (a) The seamless points and outdoor condition accuracy and (b) direction value of integration location

path recording the process until reached an outdoor location. From the tracking task, the user's current GPS location matched both factors such as recorded location path coordinates and device placement positions to ensure that the regions are the same, which were recorded in the system from the floor plans. Devices were used on different floors, but location coordinates were the same so that the system detected the floor according to both situations of placement of devices and location coordinates.

An accuracy had been practically experimented with the data set value for indoor, integration (overlapped) and outdoor regions (Fig. 8); these were related to the number of time that the signal values were received, the location coordinates either enters or exists in indoor. The accuracy of certainty value when user starts operating the system is $98.4 \%$ when moving from outdoor to indoor and from indoor moved to outdoor is $97.7 \%$ accuracy at the first floor, the cetainty value is dependent on the current positioning and reference points at the fixed points based indoor localization until coverage area of overlapped regoin to outdoor environment.

Meanwhile, integration and outdoor regions were calculated by the current positioning and the executed timing on the user's side and application processing 
period. In order to make sure that the performance of the developed system is working correctly.

The three regions such indoor, integration and outdoor areas were measured differently. The average coordinate operation ratio and received signal strength were recorded into the system, and the directional pathway was shown with the set of value of coordinate points, such as latitude and longitude of the reference points based on indoor, outdoor, integration points. The tracked location of a seamless scenario had indicated that the different localization had been combined when the user was moving from a current location into a destination with smooth transition. The user was also continuously tracked with a high accuracy in real-time.

\section{Conclusion}

In this research, propose the seamless localization routing system and specification position was based on indoor and outdoor environments; the experiment was implemented with three kinds of conditions such as indoor, integration, and outdoor regions. The utilization of the proposed system has fulfilled the requirements of the user's needs with its features which are easy to use and save the user's time. People can find specific positioning or rooms on their own with this application without asking anyone and change to other systems when they move from outdoor to indoor localization. The proposed application uses intelligence and improved localization and positioning system that is attached to the smartphone, wi-fi fingerprint, beacons, pedometer, magnetic compass, graphs, and accelerometer. The system provides guidance and highlighted map about the pathway of the desired location and provide a path of multi rooms and plot visit order based on the current location, the system integrated tracking of localization seamlessly for both of different environments, the certainly value of seamlessness terms are $98.4 \%$ accuracy at the overlapped area into indoor region which is covered from large granularity of the location context to specific positioning.

On the other hand, a seamless localization routing system provides highlighted pathway according to the user at the current position and destination. The system is a fast transition less than 5 seconds switching between outdoor to indoor environment when user starts moving from current positioning to the destination, secure and robust system, and provides authenticity to the system when integration is applied in terms of indoor tracking, overlapped, and outdoor tracking. Indoor and outdoor environments coverage using the hybrid technique with improved indoor and shortest path to navigate the path and perform indoor positioning system by this proposed seamless tracking to cover all requirements and both indoor and outdoor conditions. However, implementation and evaluation of the proposed system have some limitations when being applied to the real environment, such as signal coverage both indoor and outdoor, user's speed walking, and transaction period; all these need to have improvement in order to obtain higher performance and ensure that the system do not miss any goals. These will be also be addressed in future works to continuously improve on the current prototype with better UI (user interface) design based mobile application, aside from gaining further improvement in the real-time performance expansion of the system to a broader environment.

\section{Conflicts of Interest}

The authors declare no conflict of interest.

\section{Author Contributions}

The research was conducted by Khamla NonAlinsavath, Lukito Edi Nugroho, Widyawan Kazuhiko HAMOTO and Somphone Kanthavong; this work was carried out as a part of my Ph.D. dissertation; Khamla NonAlinsavath wrote the paper, prepared all related works and collected data from other authors. Lukito Edi Nugroho, Widyawan and Kazuhiko HAMAMOTO analyzed, revised the data and checked all content of this research; They also acted as supervisors and guided of this research work; all authors had approved the final version.

\section{Acknowledgments}

I would like to express my deepest gratitude to my advisors, and colleagues for their technical and/or material support and assistance. Universitas Gadjah Mada and Tokai University are acknowledged for their support and facilitation in this research. I would like to thank the AUN-SEED/Net (ASEAN University Network/Southeast Asia Engineering Education Development Network) Program, JICA (Japan International Cooperation Agency) Project for the financial support.

\section{References}

[1] J. Shang, S. Chen, J. Wu, and S. Yin, "ARSpy: Breaking Location-based Multi-player Augmented Reality Application for User Location Tracking", IEEE Trans. Mob. Comput., pp. 1-1, 2020. 
[2] S. Thiprak and W. Kurutach, "Ubiquitous computing technologies and Context Aware Recommender Systems for Ubiquitous Learning", In: Proc. of Electrical Engineering/Electronics, Computer, Telecommunications and Information Technology (ECTI-CON), $2015 \quad$ 12th International Conf. on, 2015, pp. 1-6.

[3] S. He and S.-G. Chan, "Wi-Fi Fingerprint-Based Indoor Positioning: Recent Advances and Comparisons", IEEE Commun. Surv. Tutor., Vol. 18, No. 1, pp. 466-490, 2016, doi: 10.1109/COMST.2015.2464084.

[4] S. Li, G. Li, L. Wang, and Y. Qin, "SLAM integrated mobile mapping system in complex urban environments", ISPRS J. Photogramm. Remote Sens., Vol. 166, pp. 316-332, 2020, doi: 10.1016/j.isprsjprs.2020.05.012.

[5] P. S. Gandodhar and S. M. Chaware, "Context Aware Computing Systems: A survey", In: Proc. of 2018 2nd International Conf. on I-SMAC (IoT in Social, Mobile, Analytics and Cloud) (ISMAC) I-SMAC (IoT in Social, Mobile, Analytics and Cloud) (I-SMAC), 2018 2nd International Conf. on, pp. 605-608, 2018.

[6] Y. Sun, J. Shang, and Y. Yang, Indoor and Outdoor Seamless Localization Method Based on GNSS and WLAN, Vol. 516. p. 1419, 2020.

[7] G. Guo, R. Chen, F. Ye, X. Peng, Z. Liu, and Y. Pan, "Indoor Smartphone Localization: A Hybrid WiFi RTT-RSS Ranging Approach", IEEE Access, Vol. 7, pp. 176767-176781, 2019, doi: 10.1109/ACCESS.2019.2957753.

[8] X. Guo, N. Ansari, L. Li, and L. Duan, "A hybrid positioning system for location-based services: Design and implementation", IEEE Commun. Mag., Vol. 58, No. 5, pp. 90-96, 2020, doi: 10.1109/MCOM.001.1900737.

[9] Y. Raja Vara Prasad and P. Rajalakshmi, "Context aware building energy management system with heterogeneous wireless network architecture", In: Proc. of Wireless and Mobile Networking Conf. (WMNC), 2013 6th Joint IFIP, pp. 1-8, 2013, doi: 10.1109/WMNC.2013.6548976.

[10] A. Basiri, P. Peltola, P. F. e Silva, E. S. Lohan, T. Moore, and C. Hill, "Indoor positioning technology assessment using analytic hierarchy process for pedestrian navigation services", In: Proc. of 2015 International Conf. on Location and GNSS (ICL-GNSS), pp. 1-6, 2015, doi: 10.1109/ICL-GNSS.2015.7217157.

[11] K. NonAlinsavath, L. E. Nugroho, W. Widyawan and K. Hamamoto., "Integration of Indoor Localization System using Wi-Fi
Fingerprint, Bluetooth Low Energy Beacon and Pedometer Based on Android Application Platform", International Journal of Intelligent Engineering and Systems, Vol. 13, No. 4, pp. 171-181, 2020, doi: 10.22266/ijies2020.0831.15.

[12] Q. Zeng, J. Wang, Q. Meng, X. Zhang, and S. Zeng, "Seamless Pedestrian Navigation Methodology Optimized for Indoor/Outdoor Detection", IEEE Sens. J., Vol. 18, No. 1, pp. 363-374, 2018, doi: 10.1109/JSEN.2017.2764509.

[13] S. Tomic, M. Beko, L. M. Camarinha-Matos, and L. B. Oliveira, "Distributed localization with complemented RSS and AOA measurements: Theory and method", Appl. Sci. Switz., Vol. 10, No. 1, 2020, doi: 10.3390/app10010272.

[14] S. Mushtaq, G. Alandjani, S. F. Abbasi, N. Abosaq, A. Akram, and S. Pervez, "Hybrid geolocation routing protocol for indoor and outdoor positioning applications", Int. J. Adv. Comput. Sci. Appl., Vol. 10, No. 7, pp. 1-7, 2019, doi: 10.14569/ijacsa.2019.0100701.

[15] S. Wagner, N. Fet, M. Handte, and P. J. Marrón, "An Approach for Hybrid Indoor/Outdoor Navigation", In: Proc. of 2017 International Conf. on Intelligent Environments (IE), pp. 3643, 2017, doi: 10.1109/IE.2017.22.

[16] X. Zhang, Q. Zeng, Q. Meng, J. Liu, and J. Wang, "Implementation of smartphone seamless positioning system based on mobile navigation electronic map", In: Proc. of 2016 Fourth International Conf. on Ubiquitous Positioning, Indoor Navigation and Location Based Services (UPINLBS), pp. 89-93, 2016, doi: 10.1109/UPINLBS.2016.7809955.

[17] Q. Zeng, J. Wang, Q. Meng, X. Zhang, and S. Zeng, "Seamless Pedestrian Navigation Methodology Optimized for Indoor/Outdoor Detection", IEEE Sens. J., Vol. 18, No. 1, pp. 363-374, 2018, doi: 10.1109/JSEN.2017.2764509.

[18] W. Jiang, Y. Li, C. Rizos, B. Cai, and W. Shangguan, "Seamless Indoor-Outdoor Navigation based on GNSS, INS and Terrestrial Ranging Techniques", J. Navig., Vol. 70, No. 6, pp. 1183-1204, 2017.

[19] Y. Gao, S. Liu, M. M. Atia, and A. Noureldin, "INS/GPS/LiDAR Integrated Navigation System for Urban and Indoor Environments Using Hybrid Scan Matching Algorithm", 2015.

[20] A. C. Eyng, O. K. Rayel, E. Oroski, and J. L. Rebelatto, "Kalman Filtering-Aided Hybrid Indoor Positioning System With Fingerprinting And Multilateration", In: Proc. of 2020 IEEE 
91st Vehicular Technology Conf (VTC2020Spring), 2020, pp. 1-5.

[21] E. Kazikli and S. Gezici, "Hybrid TDOA/RSS based localization for visible light systems," Digit. Signal Process., vol. 86, pp. 19-28, 2019, doi: https://doi.org/10.1016/j.dsp.2018.12.001.

[22] M. Phunthawornwong, E. Pengwang, and R. Silapunt, "Indoor Location Estimation of Wireless Devices Using the Log-Distance Path Loss Model”, In: Proc. of TENCON 2018 - 2018 IEEE Region 10 Conf., pp. 0499-0502, 2018. 